\title{
INVESTIGATING THE APPOINTMENT SCHEDULING SYSTEM AT DENTAL CLINIC USING GREEDY HEURISTICS
}

\author{
Wan Laailatul Hanim Mat Desa ${ }^{1}$, Rosshairy Abdul Rahman ${ }^{2}$, Lau Ying Hwei ${ }^{3}$, \\ Yong Choy Fung ${ }^{4}$ \\ 1,2,3,4 School of Quantitative Sciences, College of Arts and Sciences, University Utara \\ Malaysia, 06010 Sintok, Kedah, Malaysia. \\ *Corresponding E-mail : laailatul@uum.edu.my
}

\begin{abstract}
This study was designed to discuss the application of job shop scheduling with greedy technique to investigate the total time delayed for completing the dental treatment in the dental clinic. The aim of this study is to identify the total number of patients delayed for completing the dental treatment as scheduled per day. Besides, this study also aims to examine the solution obtained from each greedy technique. There are three greedy techniques have been employed which are the earliest due date, shortest processing time and longest processing time. The result of the investigation indicated that the techniques of the earliest due date and shortest processing time provide optimal solution with no delays. It is shows that the solution from this study can be used as a guideline of patient scheduling in any related application.
\end{abstract}

Keywords: appointment, job shop scheduling, dental clinic, number of patients delayed, total time delayed DOI: https://doi.org/10.30880/jtmb.2018.05.03.004

\subsection{Research Background}

Scheduling is an important aspect of operations control in both manufacturing and services industries. Scheduling normally used to determine the started time and the finish time for each job. If the scheduling of operations is efficient, then it will help to improve the performance of the system (Ho, Lau \& Li, 1995).

Nowadays, the most important goal in the medical sector is to improve the quality of the service, especially in dentistry. Currently, the trend of the dental care is to pursue the excellence in quality of service and best practices. In order to retain patients due to the high competition, much dental concentrate on the enhancement of service quality (Abdus-Salaam, Davis, \& De Oliveira Mota, 2009). Therefore, by the increased of the competition, the dental has to improve the service quality by reducing the waiting times for the patients. This is because most of the services provided by the dental care are homogenous, but frequently with different quality level. Thus, the dental can conduct an appointment scheduling by determining the started time, duration, and the number of appointments for a given appointment in order to manage the capacity to meet the patient's daily demand.

Dental clinic is a part of social and medical institutions, its role to provide dental care to the population and out- patient services. Normally, dental clinic provides the services of cleaning, removing teeth, filling, swollen gums, and emergency cases such as painful or accidents. Certain services only provided on a specific day such as services for cleaning provided on Sunday, Monday, and Tuesday and the service for filling provided on Wednesday and Thursday. Since the demand of the patient toward the dental services is increased, thus, this dental clinic has tried their best to give the best services to the patients. 


\subsection{Problem Statement}

A dental clinic needs to be very competitive and aggressive to improve their service quality. An initiative to solve the problem at this dental clinic have to take into account since there are still no scheduled appointments to get the dental treatment. Hence, this situation causes the dental clinic is always congested by the patient, especially on Tuesday.

The management of dental clinic seeks to improve the quality of the dental service by schedule the patient based on the appointment made by the patient. In order to schedule the appointments of the patients for the dental treatment, the expected processing time for each patient is estimated by the dentist based on their experience. The expected processing time for completing the dental treatment is a critically important since the dental treatment for the next patient will be delayed if it is violated. In directly, it could affect the satisfaction of the patient toward the dental service quality.

Therefore, this study was conducted to help the dental clinic to schedule the appointments for the patients with the minimization of the total delayed time for completing the dental treatment and the total number of patient delayed.

\subsection{Research Objectives}

This study discusses the application of the greedy heuristic technique to schedule the appointments made by the patients at a dental clinic. The purpose of conducting this study includes:

- To determine the total time delayed for completing the dental treatment.

- To identify the total number of patients delayed for completing the dental treatment as scheduled per day.

- To compare the solution of each greedy technique.

\subsection{Significance of the study}

This study will contribute to the management of a dental clinic to solve the problem of crowded in the dental clinic by appointment scheduling. Besides that, this study also enables the dental department to schedule the appointments by ordering the patients with minimum total time delays and total delayed patient for the purpose to enhance the quality of the dental service and improve the dental clinic overall operational efficiency.

\subsection{Literature Review}

The efficiency and effectiveness of running service systems are gradually receiving more attention. This is because the services in industries have become a more important in business sector. Applying the appointment scheduling rules would allow us to investigate the effectiveness. In the services system, the customer waiting times are valuable where the service productivity can be improved by using appointment scheduling rules (Klassen \& Yoogalingm, 2009).

Besides that, in the developed country, the complex hospitals require patients to make appointments in advance to get the healthcare service. This is because the complex hospital consists of many treatment clinics. Since there are many treatment clinics in the complex hospital, some patients might need multiple appointments on the same days for the related treatment clinics (Abdus-Salaam, Davis, \& De Oliveira Mota, 2009). If the patient cannot fulfill the appointments in the same day, then they might make a late cancellation or do not attend or not fulfill the appointment schedule. Due to that causes, the operational cost of the hospital will increase due to the idle time of resources and rescheduled appointment by patients. Therefore, 
the management of the complex hospital has to improve the appointment scheduling system for the enhancement of the efficiency and effectiveness operational for the complex healthcare systems.

Although there are multiple doctors in a clinic, patient more preferable seeing the same doctor rather than being assigned to the other physician randomly. Thus, the favor doctors will have full schedules. If the patient double booking the appointment, then the no-show of patients will occur (S. Ahmed \& Amagoh, 2014). In addition, the fundamental challenge for the clinic is to manage the clinic's capacity in order to meet the patients' daily demand. This involves to limit the noshow of patients rate with prescheduled appointments and to ensure there are enough of available slots for the same-day appointment request (Tennant \& Kruger, 2014). The complexity will increase if the multiple appointments are giving with the limited resources for available slots and physicians. Hence, the most challenging for the medical institutions is to schedule the appointments to fulfill the patients need and avoid the increases of the cost.

Referring to (Abdus-Salaam, Davis, \& De Oliveira Mota, 2009), block scheduling with priority (BSP) policy was developed for solving the appointment scheduling in the multi-clinic system. The specific appointment time slot reserved for the patients with priority is called as a block. For instance, the clinic opens first four timeslots for any patient and reserve the last three for patients with priority which called as a block. The result shows that the BSP policy is more suitable for the higher demand of the multi-clinic appointments system compares by the first come first serve (FCFS) policy.

Meanwhile, in order to solve the double-booking strategy, the metaheuristic is employed which included tabu search, integer programming, and neural network by (S. Ahmed \& Amagoh,2014). A minimization mathematical formulation is built to test a good solution between double bookings and block appointment scheduling. Thus, the best solution to cover the higher no-show of patient rate is by assign first two clients at the beginning of the session. This will help to infinite the clinic to serve its patient population by reducing the no-show rate.

Besides that, for the multiple appointments, Markov decision process (MDP) model is used to determine whether to accept a batch appointments request for the same day slot (Tennant \& Kruger, 2014). A batch refers to a parent with their children either one or more children. Therefore, the result shows that the dental clinic should accept the batch appointments request depend on the number of prescheduled patients and maximum backlog if the no-show rate is greater than 0.60. There are several methods have been used to solve the schedule appointment problem to allocate the suitable time for setting the appointment in healthcare system. One of the technique that also can implement to the scheduling appointment is by using greedy heuristic technique as highlighted in this study.

\subsection{Description of the Case Study}

In this study, only one dentist will handle the dental treatment for all the patients because the clinic is experiencing a shortage of dentist. The dental clinic wishes to schedule an appointment for the patient so that the patient does not waste their time waiting to get the dental treatment. The patient can make an appointment 3 days before the dental treatment either by walk-in or contact the receptionist. The patient will get to know their appointment time for the dental treatment one day before the treatment by receiving the message from the dental clinic.

Before running the appointment scheduling for the dental clinic, the management team of dental clinic has identified the expected processing time to complete the dental treatment is violating or not compare to the actual processing time for the patient to complete their dental treatment. Observation is conducted in the dental clinic on Tuesday. The data of actual processing time for each patient to complete the dental treatment is collected by observing the time of patients enter the dental treatment room and the time they leave the dental treatment 
room. Since the observation is carried out on Tuesday which only have the services of cleaning and emergency cases provided, thus, the dentist is expected that to complete the dental treatment of cleaning will take almost 10 minutes, whereas the emergency cases will take almost 15 minutes.

The assumptions of this study is the dentist is always available throughout the scheduling period. Besides that, the dentist cannot handle more than one patient at a time and each patient must spend on the dental treatment as prescribed length of time.

\subsection{Data Collection}

This study consists of two types of data which are primary data and secondary data. The primary data was the data collected through an interview session with the dentist and observation toward the service length of the patients in the dental clinic. Whereas, the secondary data for this study was the data gathered from the website about the history of the dental clinic. The early step for the data collection was carried out by an interview the dentist at the dental clinic. First, it was noted that patients typically come for the dental treatment on Tuesday. In addition, the dentist also estimated that 10 minutes is required for the cleaning service and 15 minutes for the emergency service based on their experiences.

The collection of the data was taken through all the processes at the dental clinic. The aim was to get the time between patient consecutive arrivals and the service length during the collection of the data. Thus, the collected data included:

- The time of patients enters the dental treatment room

- The time of patients out the dental treatment room

\subsection{Methodology}

The techniques used in this study is a greedy heuristic for job shop scheduling. The purpose of using this technique is to find an ordering of the patients that can minimize the total time of delays and the total number of delayed patients. The greedy heuristic technique in job shop scheduling consists of three types which are the earliest due date, shortest processing time, and longest processing time. Thus, the patients had to arrange accordingly either earliest expected processing time to complete the dental treatment or shortest actual processing time, and longest actual processing time.

As shown in Fig. 1, after the arrangement, the completing processing time and the total expected processing time for each patient were computed. The comparison will be carried out between completed processing time and the total expected processing time for each patient. If the completed processing time for each patient is greater than the total expected processing time, then the delaying of dental treatment is occurred. The total time of dental treatment delayed and the total number of patient delayed is then calculated.

There is a comparison of the total time of dental treatment delayed and the total number of the patient delayed among these three greedy heuristic techniques. One of the technique will be selected and consider as an optimal solution if it obtains a minimum total time of dental treatment delayed and a total number of delayed patient. Matlab version 7.8 was used to find the solution for all the greedy heuristic techniques. In order to measure the accuracy of the solution, QM for Windows was also employed. 


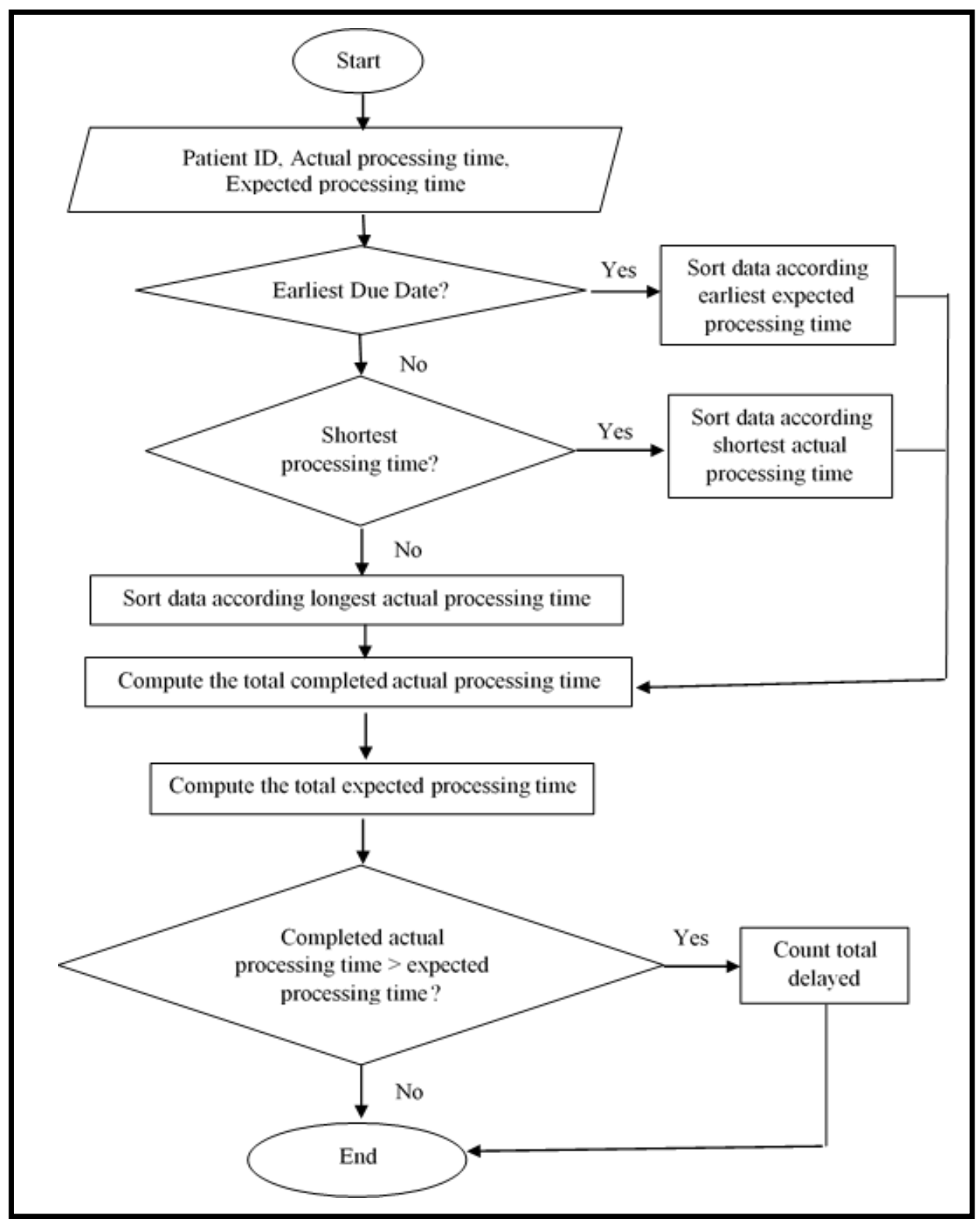

Figure 1: Flow Chart of Greedy Heuristic Technique

\subsection{Results and Discussion}

Based on Table 1, the total time delayed and the total number of patient delayed were 0 for the earliest due date and shortest processing time. The ordering of the patient for earliest due date (EDD) was patient 1 as a starting patient, follow by the patient $2,3,5,6,7,8,9,10,11,13,14$, $15,16,17,18,19,20,21,22,23,25,27,29,30,31,32,33,34,4,12,24,26$, and ended by the patient 28 . However, for the shortest processing time, the scheduling should start with patient 33 as a starting patient, follow by patient $33,1,5,11,30,32,15,31,10,16,22,2,7,8,13,18,23$, $27,6,9,14,17,19,25,20,21,29,3,34,12,28,4,26$, and ended by the patient 24 . Unfortunately, for the longest processing time, the total time delayed was 475 minutes ( 7 hours and 54 minutes) and the total number of patient delayed was 29 patients. The scheduling process was the patient 24 as a starting patient, then follow by patient $4,26,12,28,3,34,29,20,21,6,9,14,17,19,25$, $2,7,8,13,18,23,27,10,16,22,15,31,1,5,11,30,32$, and ended by patient 33 . 
Table 1: Result for Each Greedy Heuristic Technique

\begin{tabular}{llcc}
\hline \multicolumn{1}{c}{ Technique } & \multicolumn{1}{c}{ Patient Ordering } & $\begin{array}{c}\text { Total Time } \\
\text { Delay (minutes) }\end{array}$ & $\begin{array}{c}\text { Total Number of } \\
\text { Patient Delayed }\end{array}$ \\
\hline Earliest Due Date & $1,2,3,5,6,7,8,9,10,11,13,14,15,16$, & 0 & 0 \\
& $17,18,19,20,21,22,23,25,27,29,30$, & & \\
& $31,32,33,34,4,12,24,26,28$ & & \\
Shortest Processing Time & $33,1,5,11,30,32,15,31,10,16,22,2,7$, & 0 & \\
& $8,13,18,23,27,6,9,14,17,19,25,20$, & & \\
& $21,29,3,34,12,28,4,26,24$ & & \\
Longest Processing Time & $24,4,26,12,28,3,34,29,20,21,6,9,14$, & 475 & \\
& $17,19,25,2,7,8,13,18,23,27,10,16$, & & \\
& $22,15,31,1,5,11,30,32,33$ & & \\
\hline
\end{tabular}

\subsection{Conclusion and Recommendation}

As a conclusion, a different technique of ordering the appointment of the patient will have different total time delayed and the total number of patient delayed. Thus, this study found that the method of earliest due date and shortest processing time offer the minimum total time delayed and the total number of patient delayed. Hence, the dental clinic should schedule the patient by earliest due date or shortest processing time in order to get the minimum total time delayed and the total number of patient delayed to avoid unhappy patient in getting the dental treatment. For future research, more data involving two weeks scheduling could be use thus providing more valuable solution for the society and also can assist the dental clinic to be more efficient to serve their patients.

\section{Acknowledgement}

We are sincerely thanks to the dentist in the dental clinic for allowing us to have an interview and providing us the valuable information to the development of the mathematical model and complete this study. 


\section{References}

Abdus-Salaam, H., Davis, L., \& De Oliveira Mota, D. (2009). Modeling Dependent Demand Arrivals Within an Open-Access Scheduling System. TIC-STH'09: 2009 IEEE Toronto International Conference - Science and Technology for Humanity, 256-261.

Ahmed, S., \& Amagoh, F. (2014). Process Analysis and Capacity Utilization in a Dental Clinic in Kazakhstan. Competitiveness Review, 24(4), 347-356.

Harper, P., Kleinman, E., Gallagher, J., \& Knight, V. (2013). Cost-Effective Workforce Planning: Optimising the Dental Team Skill-Mix for England. Journal of Enterprise Information Management, 26(1/2), 91-108.

Ho, C., Lau, H.-S., \& Li, J. (1995). Introducing Variable-Interval Appointment Scheduling Rules in Service Systems. International Journal of Operations \& Production Management, 15, 59-68.

Klassen, K. J., \& Yoogalingam, R. (2009). Improving Performance in Outpatient Appointment Services with a Simulation Optimization Approach. Production and Operations Management, 18(4), 447-458.

Tennant, M., \& Kruger, E. (2014). Building a Stronger Child Dental Health System in Australia: Statistical Sampling Masks the Burden of Dental Disease Distribution in Australian Children. Rural and Remote Health, 14(3). 